EDO-EP-37

March, 2001

\title{
Antisymmetric Tensor Fields in the Locally Localized Gravity Models
}

\author{
Ichiro Oda f \\ Edogawa University, 474 Komaki, Nagareyama City, Chiba 270-0198, JAPAN
}

\begin{abstract}
We study the localization property of antisymmetric tensor fields in the locally localized gravity models. It is shown that all the antisymmetric tensor fields, including the vector field, in a bulk space-time are trapped on an $A d S$ brane by a gravitational interaction where the presence of the brane cosmological constant plays an important role as in the cases of the other bulk fields. The normalized zero-modes spread rather widely in extra space so small extra dimensions might be needed in order not to conflict with experiment.
\end{abstract}

\footnotetext{
${ }^{1}$ E-mail address: ioda@edogawa-u.ac.jp
} 
There has been a great deal of excitement recently over an alternative compactification scenario of space-time, where our four dimensional world emerges as a topological defect, a 3-brane, in a higher dimensional space-time [1, 2]. (This model was generalized to the case of many branes in Ref. [3].) In such 'brane world' models, it is sometimes supposed that all the familiar matter and gauge fields are constrained to live on the brane, whereas gravity is free to propagate in the whole space-time. However, this treatment is not democratic at all as long as we do not have any reasonable mechanism of confinement of the matter and gauge fields on the brane. Thus, we should take account of all the local fields as bulk fields in the 'brane world' models.

In particular, one crux in the original Randall-Sundrum model is then how to localize the bulk gauge fields on a brane by a gravitational interaction. It is well known that in the Randall-Sundrum model the photon and the Kalb-Ramond field cannot be localized on the brane [4, 5, 6].

Recently, in Refs. [7, 8] we have developed a new mechanism for the localization of the bulk fields on the brane in the locally localized gravity models [9, 10, 11, 12, 13]. In this mechanism, the presence of the brane cosmological constant plays a crucial role.

The aim of this paper is to study the localization property of the bulk antisymmetric

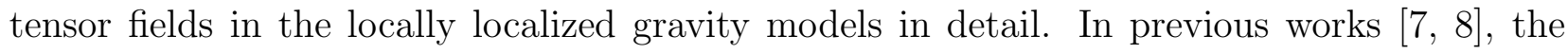
antisymmetric tensor fields were not considered explicitly. But if we regard the models at hand as coming from superstring theory a bunch of antisymmetric tensor fields are expected to emerge in the low energy field theory so it is of importance to give a full analysis for the bulk antisymmetric tensor fields. Indeed, D-branes, in which D3 brane is one of candidates as our world, carry the nontrivial charges of the R-R antisymmetric tensor fields [14]. Moreover, it is pointed out that a brane with the charge of an antisymmetric tensor field might be stable owing to the charge conservation law [15].

First, we shall start with a locally localized gravity model by Karch and Randall in five dimensions [10]. Unlike their paper, we shall make use of the formulation adopted in our previous paper [8] since not only the translation to the Karch-Randall formulation but also the generalization to higher dimensions are straightforward. The metric ansatz we take is of the form [8]:

$$
\begin{aligned}
d s_{(5)}^{2} & =g_{M N} d x^{M} d x^{N} \\
& =\mathrm{e}^{-A(r)} \hat{g}_{\mu \nu} d x^{\mu} d x^{\nu}+d r^{2} \\
& =\mathrm{e}^{-A(z)}\left(\hat{g}_{\mu \nu} d x^{\mu} d x^{\nu}+d z^{2}\right),
\end{aligned}
$$

where $M, N, \ldots$ denote five-dimensional space-time indices and $\mu, \nu, \ldots$ four-dimensional brane ones. The metric over the brane $\hat{g}_{\mu \nu}$ denotes the four-dimensional anti-de Sitter metric. Moreover, $e^{-A(r)}, e^{-A(z)}$ and the relation between the 'radial' $r$-coordinates and the 'conformal' $z$-coordinates are given by

$$
\begin{aligned}
\mathrm{e}^{-A(r)} & =\cosh ^{2} \omega r \\
\mathrm{e}^{-A(z)} & =\frac{1}{\sin ^{2} \omega z}
\end{aligned}
$$




$$
\mathrm{e}^{\omega r}=\tan \frac{1}{2} \omega z
$$

where $\omega$ denotes $\sqrt{\frac{-\Lambda}{6}}$ with $\Lambda$ being the negative bulk cosmological constant. The brane cosmological constant $\Lambda_{A d S}$ is then expressed in terms of the bulk cosmological constant $\Lambda$ via $\Lambda_{A d S}=\frac{1}{2} \Lambda$. In other words, this model describes an $A d S_{4}$ brane sitting at the origin $r=0$ in $A d S_{5}$. Notice that since the 'radial' coordinate $r$ runs from 0 to $\infty$, this relation yields the range of $z$, which is $\frac{\pi}{2 \omega} \leq z \leq \frac{\pi}{\omega}$.

In what follows, we shall consider from a 0-form potential, i.e., a massless real scalar field, to a 3 -form potential in five dimensions. A 4-form potential is non-dynamical field, for which we shall not take into consideration. The action of a 0 -form potential $\Phi$ is given by

$$
S_{0}=-\frac{1}{2} \int d^{5} x \sqrt{-g} g^{M_{1} N_{1}} F_{M_{1}} F_{N_{1}}
$$

where $F_{M}=\partial_{M} \Phi$. The equation of motion becomes

$$
\partial_{M_{1}}\left(\sqrt{-g} g^{M_{1} N_{1}} F_{N_{1}}\right)=0 .
$$

Let us look for a solution with the form of

$$
\Phi\left(x^{M}\right)=\phi\left(x^{\mu}\right) u(z)
$$

where we assume the equation of motion for the brane field $\hat{\nabla}^{\mu} f_{\mu}=0$ with being $f_{\mu}=\partial_{\mu} \phi$. With the ansatz (5), Eq. (4) reduces to a single differential equation for $u(z)$ :

$$
\partial_{z}\left(\mathrm{e}^{-\frac{3}{2} A(z)} \partial_{z} u\right)=0
$$

The general solution is easily found to be

$$
u(z)=-\frac{\alpha}{3 \omega} \cos \omega z\left(\sin ^{2} \omega z+2\right)+\beta
$$

where $\alpha, \beta$ are integration constants.

Plugging the form of a solution (5) into the starting action, the action can be cast to

$$
\begin{aligned}
S_{0}^{(0)} & =-\frac{1}{2} \int d^{5} x \sqrt{-g} g^{M_{1} N_{1}} F_{M_{1}}^{(0)} F_{N_{1}}^{(0)} \\
& =-\frac{1}{2} \int d^{4} x \sqrt{-\hat{g}} \int d z \mathrm{e}^{-\frac{3}{2} A(z)}\left[u^{2}(z) \hat{g}^{\mu \nu} \partial_{\mu} \phi \partial_{\nu} \phi+\left(\partial_{z} u\right)^{2} \phi^{2}\right] .
\end{aligned}
$$

Provided that the general solution (7) is inserted to this action, it turns out that the integral

$$
I_{1}=\int_{\frac{\pi}{2 \omega}}^{\frac{\pi}{\omega}} d z \mathrm{e}^{-\frac{3}{2} A(z)} u^{2}(z)
$$


is in general divergent, but only when $\beta=-\frac{2 \alpha}{3 \omega}$ it becomes strictly finite. Then, the zero-mode $u(z)$ and $I_{1}$ are respectively given by

$$
\begin{aligned}
u(z) & =-\frac{\alpha}{3 \omega}\left[\cos \omega z\left(\sin ^{2} \omega z+2\right)+2\right], \\
I_{1} & =\frac{\alpha^{2}}{9 \omega^{3}}\left(\frac{1}{3}+4 \log 2\right) .
\end{aligned}
$$

The fact that $I_{1}$ is finite shows that a 0 -form potential in a bulk is trapped on an $A d S_{4}$ brane by a gravitational interaction.

After evaluating the second integral over $z$ in (8), we obtain

$$
S_{0}^{(0)}=-\frac{1}{2} \int d^{4} x \sqrt{-\hat{g}}\left[\frac{\alpha^{2}}{9 \omega^{3}}\left(\frac{1}{3}+4 \log 2\right) \hat{g}^{\mu \nu} \partial_{\mu} \phi \partial_{\nu} \phi+\frac{2 \alpha^{2}}{3 \omega} \phi^{2}\right] .
$$

To make the kinetic term take a canonical form, let us redefine the field as $\frac{\alpha}{3 \omega^{\frac{3}{2}}} \sqrt{\frac{1}{3}+4 \log 2} \phi \rightarrow$ $\phi$. Then, we have

$$
S_{0}^{(0)}=-\frac{1}{2} \int d^{4} x \sqrt{-\hat{g}}\left[\hat{g}^{\mu \nu} \partial_{\mu} \phi \partial_{\nu} \phi+\frac{6 \omega^{2}}{\frac{1}{3}+4 \log 2} \phi^{2}\right] .
$$

Since the brane cosmological constant $\Lambda_{A d S}$ is required to be tiny in order to make contact with observations, the mass of a scalar field on a brane must be very small.

At this stage, let us examine the zero mode $u(z)$ in more detail. First, note that $u(z)$ in (10) satisfies the Dirichlet boundary condition at $z=\frac{\pi}{\omega}$ where it is known that the gravitational potential becomes infinity [10]. Second, we see that the normalized wave function in the $\hat{g}_{M N}$ space takes the form

$$
\begin{aligned}
\hat{u}(z) & =-\frac{1}{\sqrt{I_{1}}} \mathrm{e}^{-\frac{3}{4} A(z)} u(z) \\
& =\sqrt{\frac{\omega}{\frac{1}{3}+4 \log 2}} \frac{-\cos ^{3} \omega z+3 \cos \omega z+2}{(\sin \omega z)^{\frac{3}{2}}} \\
& =\sqrt{\frac{2 \omega}{\frac{1}{3}+4 \log 2} \frac{3 \mathrm{e}^{-\omega r}+\mathrm{e}^{-3 \omega r}}{\left(\mathrm{e}^{\omega r}+\mathrm{e}^{-\omega r}\right)^{\frac{3}{2}}}} \\
& \approx 3 \sqrt{\frac{2 \omega}{\frac{1}{3}+4 \log 2}} \mathrm{e}^{-\frac{5}{2} \omega r}
\end{aligned}
$$

where Eq. (2) was utilized and the last expression holds for $r \gg 1$. Eq. (13) implies that the wave function is localized near the origin $r=0$. However, there is a caveat. Because of $\omega \ll 1$ this wave function spreads rather widely in a bulk space, so we might require the extra space to be small enough to be consistent with observations. This 'small extra dimensions' scenario might shed light on the conventional Kaluza-Klein compactification idea. Namely, in the model at hand the size of extra dimension is dependent on the size of the brane cosmological 
constant. As is well known that observations suggest a very tiny cosmological constant, extra dimension should be very small. At present it is not clear whether this new scenario is really a viable one, but it is of interest to note that the size of extra space is determined by that of the cosmological constant on our brane. As a final remark, it is known that traceless, transverse graviton modes in general obey the same equations of motion as a massless scalar field in a curved background. Therefore, the present result tells us that the graviton is also trapped on an $A d S_{4}$ brane.

Next, we shall turn to a 1 -form potential, that is, the massless $U(1)$ gauge field [7]. The path of arguments is very similar to the case of a 0 -form potential. The action is

$$
S_{1}=-\frac{1}{4} \int d^{5} x \sqrt{-g} g^{M_{1} N_{1}} g^{M_{2} N_{2}} F_{M_{1} M_{2}} F_{N_{1} N_{2}}
$$

where $F_{M N}=\partial_{M} A_{N}-\partial_{N} A_{M}$. The equations of motion become

$$
\partial_{M_{1}}\left(\sqrt{-g} g^{M_{1} N_{1}} g^{M_{2} N_{2}} F_{N_{1} N_{2}}\right)=0 .
$$

After taking the gauge condition $A_{z}\left(x^{M}\right)=0$, we search for a solution with the form of

$$
A_{\mu}\left(x^{M}\right)=a_{\mu}\left(x^{\lambda}\right) u(z)
$$

where we assume the equations of motion on a brane $\hat{\nabla}^{\mu} a_{\mu}=\hat{\nabla}^{\mu} f_{\mu \nu}=0$ with the definition of $f_{\mu \nu}=\partial_{\mu} a_{\nu}-\partial_{\nu} a_{\mu}$. With this ansatz, Eq. (15) also reduces to a single differential equation for $u(z)$ :

$$
\partial_{z}\left(\mathrm{e}^{-\frac{1}{2} A(z)} \partial_{z} u\right)=0
$$

whose general solution is given by

$$
u(z)=-\frac{\alpha}{\omega} \cos \omega z+\beta
$$

where $\alpha, \beta$ are integration constants.

Plugging this solution into the classical action (14), selecting $\beta=-\frac{\alpha}{\omega}$ which gives rise to only the finite kinetic term, and evaluating the integrals over $z$, the action takes the form

$$
\begin{aligned}
S_{1}^{(0)} & =-\frac{1}{4} \int d^{5} x \sqrt{-g} g^{M_{1} N_{1}} g^{M_{2} N_{2}} F_{M_{1} M_{2}}^{(0)} F_{N_{1} N_{2}}^{(0)} \\
& =-\frac{1}{4} \int d^{4} x \sqrt{-\hat{g}}\left[\frac{\alpha^{2}}{\omega^{3}}(-1+2 \log 2) \hat{g}^{\mu_{1} \nu_{1}} \hat{g}^{\mu_{2} \nu_{2}} f_{\mu_{1} \mu_{2}} f_{\nu_{1} \nu_{2}}+\frac{2 \alpha^{2}}{\omega} \hat{g}^{\mu_{1} \nu_{1}} a_{\mu_{1}} a_{\nu_{1}}\right]
\end{aligned}
$$

Redefining $\frac{\alpha}{\omega^{\frac{3}{2}}} \sqrt{-1+2 \log 2} a_{\mu} \rightarrow a_{\mu}$, the above action can be rewritten as

$$
S_{1}^{(0)}=-\frac{1}{4} \int d^{4} x \sqrt{-\hat{g}}\left[\hat{g}^{\mu_{1} \nu_{1}} \hat{g}^{\mu_{2} \nu_{2}} f_{\mu_{1} \mu_{2}} f_{\nu_{1} \nu_{2}}+\frac{2 \omega^{2}}{-1+2 \log 2} \hat{g}^{\mu_{1} \nu_{1}} a_{\mu_{1}} a_{\nu_{1}}\right]
$$


As stressed in Ref. [0], the massless condition of 'photon' on a brane forces us to choose $\omega \approx 0$ as expected from a small value of the cosmological constant.

Let us study the zero-mode $u(z)$. With a specific choice $\beta=-\frac{\alpha}{\omega}, u(z)$ becomes

$$
u(z)=-\frac{2 \alpha}{\omega} \cos ^{2} \frac{1}{2} \omega z
$$

which also satisfies the Dirichlet boundary condition at $z=\frac{\pi}{\omega}[7,8$. And the normalized wave function is calculated as

$$
\begin{aligned}
\hat{u}(z) & =-\frac{1}{\sqrt{I_{1}}} \mathrm{e}^{-\frac{1}{4} A(z)} u(z) \\
& =\sqrt{\frac{\omega}{-1+2 \log 2}} \frac{\cos \omega z+1}{(\sin \omega z)^{\frac{1}{2}}} \\
& =\sqrt{\frac{2 \omega}{-1+2 \log 2}} \frac{\mathrm{e}^{-\omega r}}{\left(\mathrm{e}^{\omega r}+\mathrm{e}^{-\omega r}\right)^{\frac{1}{2}}} \\
& \approx \sqrt{\frac{2 \omega}{-1+2 \log 2}} \mathrm{e}^{-\frac{3}{2} \omega r},
\end{aligned}
$$

where this time $I_{1}=\frac{\alpha^{2}}{\omega^{3}}(-1+2 \log 2)$ and the last expression also holds for $r \gg 1$. Eq. (22) again means that the wave function is localized near the origin $r=0$ but spreads rather widely in a bulk space. It is remarkable that both the 0 -form potential and the 1 -form potential share the same features and demand the 'small extra dimensions' scenario. Incidentally, $\hat{u}(z)$ in (13) and (22) also satisfies the Dirichlet boundary condition at $z=\frac{\pi}{\omega}$.

We are now ready to consider a 2 -form potential, in other words, the Kalb-Ramond field. In five dimensions, the 2 -form potential is dual to the 1 -form potential, so it is expected that the 2-form potential is also trapped on a brane as in the 1-form one. Below we shall explicitly show that this is indeed the case. The arguments proceed in the same way as in the 0-form and 1 -form potentials. The classical action is given by

$$
S_{2}=-\frac{1}{12} \int d^{5} x \sqrt{-g} g^{M_{1} N_{1}} g^{M_{2} N_{2}} g^{M_{3} N_{3}} F_{M_{1} M_{2} M_{3}} F_{N_{1} N_{2} N_{3}}
$$

where $F_{M N P}=3 \partial_{[M} A_{N P]}=\partial_{M} A_{N P}+\partial_{N} A_{P M}+\partial_{P} A_{M N}$. The equations of motion are

$$
\partial_{M_{1}}\left(\sqrt{-g} g^{M_{1} N_{1}} g^{M_{2} N_{2}} g^{M_{3} N_{3}} F_{N_{1} N_{2} N_{3}}\right)=0 \text {. }
$$

The action (23) has the following gauge symmetries and the first-stage off-shell reducible symmetry 16

$$
\begin{aligned}
\delta A_{M N} & =\partial_{M} \varepsilon_{N}-\partial_{N} \varepsilon_{M}, \\
\delta \varepsilon_{M} & =\partial_{M} \varepsilon,
\end{aligned}
$$

so we can take the gauge conditions $A_{M z}=0$. (Note that the number of degrees of freedom associated with the symmetries (25) is $5-1=4$, which exactly coincides with the number of gauge conditions $A_{M z}=0$.) The natural ansatz for a solution is

$$
A_{\mu \nu}\left(x^{M}\right)=a_{\mu \nu}\left(x^{\lambda}\right) u(z),
$$


where the equations on a brane $\hat{\nabla}^{\mu} a_{\mu \nu}=\hat{\nabla}^{\mu} f_{\mu \nu \rho}=0$ with the definition of $f_{\mu \nu \rho}=3 \partial_{[\mu} a_{\nu \rho]}$ are imposed. With this ansatz, Eq. (24) also reduces to a single differential equation for $u(z)$ :

$$
\partial_{z}\left(\mathrm{e}^{\frac{1}{2} A(z)} \partial_{z} u\right)=0
$$

At this stage, notice the similarity of this equation to the other cases treated thus far. Given a p-form potential, the corresponding equation generally takes the form

$$
\partial_{z}\left(\mathrm{e}^{-\frac{3-2 p}{2} A(z)} \partial_{z} u\right)=0 .
$$

This regularity stems from the number of $g^{M N}$ in the equations of motion, or equivalently in the action. In the 2 -form potential, compared to the lower form potentials, one notable change has happened. Namely, the sign of the coefficient in front of $A(z)$ in the exponential has become positive in the present case, while negative in 0 -form and 1-form potentials. Due to this change, it turns out that we cannot find a normalizable zero-mode $u(z)$ with the nontrivial dependence of a fifth dimension $z$. The only normalizable zero-mode is given by the constant mode:

$$
u(z)=u_{0}=\text { const. }
$$

Substituting this solution into the action (23), the action reduces to the form

$$
S_{2}^{(0)}=-\frac{1}{12} \frac{u_{0}^{2}}{\omega} \int d^{4} x \sqrt{-\hat{g}} \hat{g}^{\mu_{1} \nu_{1}} \hat{g}^{\mu_{2} \nu_{2}} \hat{g}^{\mu_{3} \nu_{3}} f_{\mu_{1} \mu_{2} \mu_{3}} f_{\nu_{1} \nu_{2} \nu_{3}}
$$

Note that the constancy of the zero-mode leads to the vanishing mass term.

It is natural to ask ourselves if there is a critical difference between a p-form and its dual (3-p)-form. For instance, at first sight, the form of the zero-mode $u(z)$ appears to be different between the two forms. But this is an illusion. Actually, it is the normalized zero-mode that we should pay attention. In the case at hand, it is given by

$$
\begin{aligned}
\hat{u}(z) & =\frac{1}{\sqrt{I_{1}}} \mathrm{e}^{\frac{1}{4} A(z)} u(z) \\
& =\sqrt{\omega}(\sin \omega z)^{\frac{1}{2}} \\
& =\sqrt{2 \omega} \frac{1}{\left(\mathrm{e}^{\omega r}+\mathrm{e}^{-\omega r}\right)^{\frac{1}{2}}} \\
& \approx \sqrt{2 \omega} \mathrm{e}^{-\frac{1}{2} \omega r},
\end{aligned}
$$

where $I_{1}$ is defined as $\frac{u_{0}^{2}}{\omega}$. As before, this normalized wave function indeed satisfies the Dirichlet boundary condition at $z=\frac{\pi}{\omega}$ and has an exponentially decreasing form for $r \gg 1$.

The remaining form is a 3 -form potential, for which we shall now study. The result is very similar to the case of a 2 -form potential, so we shall touch on this case. The classical action of the 3 -form potential is

$$
S_{3}=-\frac{1}{48} \int d^{5} x \sqrt{-g} g^{M_{1} N_{1}} g^{M_{2} N_{2}} g^{M_{3} N_{3}} g^{M_{4} N_{4}} F_{M_{1} M_{2} M_{3} M_{4}} F_{N_{1} N_{2} N_{3} N_{4}},
$$


where $F_{M N P Q}=4 \partial_{[M} A_{N P Q]}=\partial_{M} A_{N P Q}-\partial_{N} A_{M P Q}+\partial_{P} A_{M N Q}-\partial_{Q} A_{M N P}$. The equations of motion are then

$$
\partial_{M_{1}}\left(\sqrt{-g} g^{M_{1} N_{1}} g^{M_{2} N_{2}} g^{M_{3} N_{3}} g^{M_{4} N_{4}} F_{N_{1} N_{2} N_{3} N_{4}}\right)=0 .
$$

Taking the gauge conditions $A_{M N z}=0$ ?, and the ansatz

$$
A_{\mu \nu \rho}\left(x^{M}\right)=a_{\mu \nu \rho}\left(x^{\lambda}\right) u(z)
$$

with the equations on a brane $\hat{\nabla}^{\mu} a_{\mu \nu \rho}=\hat{\nabla}^{\mu} f_{\mu \nu \rho \sigma}=0$ where $f_{\mu \nu \rho \sigma}=4 \partial_{[\mu} a_{\nu \rho \sigma]}$, Eq. (33) becomes

$$
\partial_{z}\left(\mathrm{e}^{\frac{3}{2} A(z)} \partial_{z} u\right)=0
$$

As a normalizable solution, we also have to select the solution (29), from which the classical action can be written as

$$
S_{3}^{(0)}=-\frac{1}{48} \frac{2}{3} \frac{u_{0}^{2}}{\omega} \int d^{4} x \sqrt{-\hat{g}} \hat{g}^{\mu_{1} \nu_{1}} \hat{g}^{\mu_{2} \nu_{2}} \hat{g}^{\mu_{3} \nu_{3}} \hat{g}^{\mu_{4} \nu_{4}} f_{\mu_{1} \mu_{2} \mu_{3} \mu_{4}} f_{\nu_{1} \nu_{2} \nu_{3} \nu_{4}}
$$

Then the normalized wave function is of form

$$
\begin{aligned}
\hat{u}(z) & =\sqrt{\frac{3 \omega}{2}}(\sin \omega z)^{\frac{3}{2}} \\
& =\sqrt{12 \omega} \frac{1}{\left(\mathrm{e}^{\omega r}+\mathrm{e}^{-\omega r}\right)^{\frac{3}{2}}} \\
& \approx \sqrt{12 \omega} \mathrm{e}^{-\frac{3}{2} \omega r}
\end{aligned}
$$

Again, this normalized wave function indeed satisfies the Dirichlet boundary condition at $z=\frac{\pi}{\omega}$ and has an exponentially decreasing form $r \gg 1$.

Thus far, we have studied p-form potentials $(p=0,1,2,3)$ in five dimensions. This analysis can be extended to p-form potentials in higher dimensions in a perfectly analogous manner. Recently, we have constructed locally localized gravity models in higher dimensions 8] where it has been pointed out that there is a nontrivial higher-dimensional extension of Karch-Randall model [10]. (There is also a trivial extention of Karch-Randall model in higher dimensions, where the physical features of the brane is the same as the Karch-Randall model so we will not consider the trivial extension in this paper.) In the nontrivial model, an $A d S_{4}$ brane is located at the origin $r=0$ in a general $D$-dimensional space-time with negative cosmological constant. The line element has the following form in the 'radial' $r$-coordinates and the 'conformal' $z$-coordinates

$$
\begin{aligned}
d s_{(D)}^{2} & =g_{M N} d x^{M} d x^{N} \\
& =\mathrm{e}^{-A(r)} \hat{g}_{\mu \nu} d x^{\mu} d x^{\nu}+d r^{2}+R_{0}^{2} \mathrm{e}^{-A(r)} d \Omega_{n-1}^{2} \\
& =\mathrm{e}^{-A(z)}\left(\hat{g}_{\mu \nu} d x^{\mu} d x^{\nu}+d z^{2}+R_{0}^{2} d \Omega_{n-1}^{2}\right),
\end{aligned}
$$

\footnotetext{
${ }^{2}$ These gauge conditions fix gauge symmetries, 1st-stage, and 2nd-stage reducible symmetries [16], which are given by $\delta A_{M N P}=\partial_{[M} \varepsilon_{N P]}, \delta \varepsilon_{M N}=\partial_{[M} \varepsilon_{N]}, \delta \varepsilon_{M}=\partial_{M} \varepsilon$.
} 
where $\mathrm{e}^{-A(r)}$ and $\mathrm{e}^{-A(z)}$ are given in Eq. (2). Here $\omega, R_{0}$, and the brane cosmological constant $\Lambda_{A d S}$ are expressed in terms of the bulk cosmological constant $\Lambda$ and the vacuum expectation value $\eta$ of scalar fields as follows:

$$
\begin{aligned}
\omega & =\sqrt{\frac{-2 \Lambda}{(D-1)(D-2)}}, \\
R_{0}^{2} & =\frac{1}{2 \Lambda}(D-2)\left(n-2-\kappa_{D}^{2} \eta^{2}\right), \\
\Lambda_{A d S} & =\frac{2}{D-1} \Lambda,
\end{aligned}
$$

where $n$ and $\kappa_{D}$ denote the number of extra dimensions and the $D$-dimensional gravitational constant, respectively.

In particular, we have studied the localization property of p-form potentials in the case of $D=6, n=2$, that is, a string-like defect model [5]. (Note that the analysis becomes more complicated as the number of $D$ gets large.) The results are very similar to the case of an $A d S_{4}$ brane in $A d S_{5}$ as shown so far, so we will not expose the detailed calculations any longer. The only difference we should mention occurs in the case of a 2-form, which is self-dual in six dimensions. Actually, provided that we start with the action (23) in six dimensions and assume a simple form of a solution $A_{\mu}\left(x^{M}\right)=a_{\mu}\left(x^{\lambda}\right) u(z) v(\theta), A_{z}\left(x^{M}\right)=A_{\theta}\left(x^{M}\right)=0$ where $\theta$ is a sixth dimension denoting the angular variable in extra space, Eq. (27) is changed to $\partial_{z}^{2} u=0$ where we have considered the zero angular momentum, that is, $\partial_{\theta}^{2} v=0$, for simplicity. Note that owing to the self-duality the exponential factor including $A(z)$ has disappeared. The normalizable solution is given by $u(z)=u_{0}$. Then, the normalized wave

function becomes $\hat{u}(z)=\sqrt{\frac{2 \omega}{\pi}}$, so it neither satisfies the Dirichlet boundary condition at $z=\frac{\pi}{\omega}$ nor has an exponential damping factor. However, there is a loophole in this argument. It is well known that there does not exist a covariant action for the self-dual 2-form in six dimensions [17], so we cannot start with the action (23) in the case at hand. In other words, we cannot deal with the self-dual forms within the present approach.

In conclusion, we have studied the localization property of p-form potentials on an $A d S_{4}$ brane in the locally localized gravity models. Although we have shown that all the p-form potentials are trapped on the brane, the corresponding wave functions spread rather widely in a bulk space-time when the brane cosmological constant is small. This fact seems to suggest that extra space is small enough not to conflict with observations. It is of interest that the size of extra dimensions is related to that of the cosmological constant. This might shed new light on both the cosmological constant problem and the compactification scenario.

\section{References}

[1] L. Randall and R. Sundrum, Phys.Rev.Lett. 83 (1999) 3370, hep-th/9905221. 
[2] L. Randall and R. Sundrum, Phys.Rev.Lett. 83 (1999) 4690, hep-th/9906064.

[3] I. Oda, Phys.Lett. B480 (2000) 305, hep-th/9908104; Phys.Lett. B472 (2000) 59, hepth/9909048.

[4] B. Bajc and G. Gabadadze, Phys.Lett.B474 (2000) 282, hep-th/9912232.

[5] I. Oda, Phys.Lett. B496 (2000) 113, hep-th/0006203; Phys.Rev. D62 (2000) 126009, hep-th/0008012.

[6] I. Oda, "Concise Encyclopedia on Supersymmetry and Noncommutative Structures in Mathematics and Physics", edited by J. Bagger, S. Duplij and W. Siegel, Kluwer Academic Publishers, Dordrecht (in press), hep-th/0009074 and references therein.

[7] I. Oda, hep-th/0012013, to appear in Phys.Lett.B.

[8] I. Oda, hep-th/0102147.

[9] I.I. Kogan, S. Mouslopoulos and A. Papazoglou, hep-th/0011141.

[10] A. Karch and L. Randall, hep-th/0011156.

[11] A. Miemiec, hep-th/0011160.

[12] M.D. Schwartz, hep-th/0011177.

[13] M. Tachibana, hep-th/0101185.

[14] J. Polchinski, "Tasi Lectures on D-branes", hep-th/9611050.

[15] T. Gherghetta, E. Roessl and M. Shaposhnikov, Phys.Lett.B491 (2000) 353, hepth/0006251.

[16] I.A. Batalin and G.A. Vilkovisky, Phys.Lett. B102 (1981) 27; Phys.Rev. D28 (1983) 2567.

[17] N. Marcus and J.H. Schwarz, Phys.Lett.B115 (1982) 111. 